\title{
2008 Summer Leadership and Research Program Abstracts
}

\author{
The following abstracts were prepared by students participating in the \\ 2008 Summer Leadership and Research Program at the Ontario Veterinary College.
}

\section{Measurement of ketosis as a risk factor for uterine disease in dairy cows \\ J. Hubbard, J. Dubuc and S. LeBlanc \\ Department of Population Medicine, Ontario Veterinary College}

The first objective of this field study was to assess the effect of storage conditions on the accuracy of a commonly used cow-side diagnostic test for ketosis. Blood and milk samples $(n=352)$ were collected from cows of all parities between 2 and 25 DIM from one Ontario dairy herd. Blood samples were centrifuged, separated and frozen within 4 hours of collection. The frozen samples were submitted to the Animal Health Laboratory at the University of Guelph for determination of serum B-hydroxybutyrate (BHB) concentration which was considered the gold standard. Milk samples were cooled immediately following collection and tested within 4 hours using KetoTest milk strips that had been stored at $21^{\circ} \mathrm{C}$ for 0 (control), 612 and 18 weeks respectively. Subclinical ketosis was defined as a serum BHB concentration of $\geq 1400 \mu \mathrm{mol} / \mathrm{L}$. The incidence of subclinical ketosis in cows was $10.7 \%$. The kappa coefficient for agreement between the control KetoTest and the test groups was $>0.95$ in all three cases. These results suggest that storage at $21^{\circ} \mathrm{C}$ for up to 18 weeks has little effect on the accuracy of the KetoTest. The second objective was to determine if an association exists between ketosis and uterine disease in postpartum dairy cows. Holstein cows of all parities from two Ontario herds and one New York herd were enrolled $(n=303)$. Blood was collected at 4,11 , and $18( \pm 3)$ DIM and was processed in the same manner as previously described. Presence of metritis, clinical endometritis and subclinical endometritis were determined based on clinical signs, the use of the Metricheck device, and the Cytobrush technique, respectively. Among 272 cows, those with BHB $\geq$ $1000 \mu \mathrm{mol} / \mathrm{L}$ did not have a higher risk of metritis than those with BHB levels below the cut-point (odds ratio $(\mathrm{OR})=0.51$, $\{95 \%$ CI 0.2-1.8, $\mathrm{P}=0.43])$. Similarly, cows with BHB $\geq$ $1000 \mu \mathrm{mol} / \mathrm{L}$ did not have a higher risk of clinical endometritis $(\mathrm{n}=272)(\mathrm{OR}=0.8,\{95 \%$ CI $0.3-2.0, \mathrm{P}=0.63])$. Cows with $\mathrm{BHB} \geq 1000 \mu \mathrm{mol} / \mathrm{L}$ had a higher risk of subclinical endometritis than those with BHB values below the cut-point $(\mathrm{OR}=2.5,\{95 \%$ CI $0.5-13)$, however these findings were not statistically significant $(\mathrm{P}=0.36)$ given the small sample size $(n=50)$. The results of this study indicate that there is no association between ketosis and metritis or clinical endometritis. In contrast, the results suggest a possible relationship between ketosis and subclinical endometritis, however these results are limited due to the small sample size.

\section{A longitudinal study of MRSA in pigs}

A.W. Zwambag, R.M. Friendship, J.S. Weese

Department of Population Medicine, Ontario Veterinary College

Methicillin-resistant Staphylococcus aureus (MRSA) are a group of multi-drug resistant Gram-positive bacteria that are of major concern to the medical community. Recent studies in Europe and Canada have shown pigs to be a major reservoir for MRSA. Transfer from pig farmers to their family and the community could have serious implications to general public health. The goal of this project was to determine at what point in the pigs life they become colonized with MRSA and to attempt to identify what risk factors contributed to colonization. Individually identified piglets from 10 sows of known MRSA status were sampled at $1,3,7,14,21,28,42,56$ and 70 days to determine the presence or absence of MRSA. Survival analysis was performed to test for the difference in the MRSA colonization times in the piglets from MRSA positive and negative sows. The hazard functions were significantly different from one another indicated by the Cox test for equality of survivor functions $(\mathrm{P}=0.0007)$. Also, by logistic regression after controlling for repeated measures within piglet, sow and group membership, the odds of a piglet testing positive for MRSA on day 28 was 1.35 times higher if it came from a MRSA positive sow than if it came from a negative sow $(\mathrm{P}=0.006)$. The rate of change in the probability of a piglet becoming MRSA positive over the length on the trial was not significantly different between piglets of MRSA positive and negative sows. This work suggests that piglets are likely getting infected from other sources in addition to the dam-piglet route which has implications on prevention strategies. 


\section{mRNA stability and the role of AU-rich elements}

J. Mortlock, J. LaMarre

Department of Biomedical Sciences, Ontario Veterinary College

Messenger RNA is translated into protein in the cell cytoplasm, where it is prone to being degraded by endogenous nucleases. mRNA degradation occurs at a specific rate that is determined by characteristics of the transcript itself and the type and state of the cell in which it is expressed. Some genes with highly variable expression patterns, such as cytokines, hormones and transcription factors, need a secondary system that modulates stability. In the 3' UTR of these mRNAs, there are specific regions called AU-Rich Elements (AREs). These regions contain many adenine and uracil residues, typically as the AUUUA pentamer. AREs are bound by proteins called AU-Rich Element Binding Proteins (ARE-BPs), which appear to modify how well nucleases in the cytoplasm can bind to the mRNA. ARE-BPs can thus change the "half-life" of the mRNA molecule by dramatically altering nuclease degradation. This allows for a more refined control over gene expression for the genes that are rapidly modulated: if rapid down-regulation is required, transcription can be stopped and destabilizing ARE-BPs effectively help clear the cytoplasm of any pre-existing mRNA, thus effecting a more rapid change. Alternatively, stabilizing ARE-BPs prolong the time during which an mRNA molecule is available for translation into protein, allowing an increase in gene expression without changing transcription rate. As many diseases result from, or are made worse by, abnormalities in the pattern and degree of gene expression there exists a clear need to further investigate this important mechanism of cellular control.

\section{Trends in avian botulism Type $\mathbf{E}$ in Anatidae, Gaviidae, and Laridae \\ T.D. Procter ${ }^{1}$, D.L. Pearl ${ }^{1}$, I.K. Barker ${ }^{2}$, G.D. Campbell ${ }^{2}$ \\ Departments of Population Medicine and ${ }^{1}$ Pathobiology, Ontario Veterinary College, Canadian Cooperative Wildlife Health Centre (CCWHC)}

In recent years, there have been anecdotal accounts that botulism type E in waterfowl, including the Anatidae (duck), Gaviidae (loon), and Laridae (gull) families, is changing in terms of the spatial and temporal distribution of outbreak incidents. A database maintained by the Canadian Cooperative Wildlife Health Centre was used to obtain data on botulism incidents in Ontario involving waterfowl from 2002-2007. Multivariable linear regression models were constructed to determine if there were significant trends in the location and/or time of botulism incidents within a summer for each family and by year. Year and family were both statistically significant variables associated with the latitude and longitude of incidents of botulism. Incidents involving Laridae were located farther north and east than incidents involving Gaviidae and Anatidae. In addition, the incidents appeared to move in a north-eastern direction between 2002 and 2007. There was also a statistically significant relationship of family and year, and an interaction effect between family and year, associated with the day of occurrence of botulism incidents in Laridae. Botulism incidents started earlier, relative to other families, and through 2002-2007 occurred later in the year. The northeastern movement of incidents may reflect distributional changes of invasive species that are anecdotally associated with botulism, and/or increased monitoring on Lake Ontario over the past few years. Nesting and migration habits may account for the family differences in the location and time of incidents. Further research into the cause, control, and trends of outbreaks may influence wildlife management and inform ecological patterns.

\section{Infection prevention and control best practices in small animal veterinary clinics}

J. Montgomery

Department of Pathobiology, Ontario Veterinary College

The Canadian Committee on Antibiotic Resistance (CCAR) has sponsored the development of best practices for asepsis and hygiene for human healthcare facilities and recognizes this need in veterinary medicine. Veterinary facilities face many of the same challenges that human healthcare facilities encounter. Hospital-acquired infections (HAIs) and outbreaks of HAIs can have a significant impact on patients, their owners and veterinary personnel, and many of the most important HAIs in human hospitals are emerging in veterinary hospitals. The close contact between most people and their pets allows for transmission of infectious agents between humans and animals, in both directions. Veterinary clinics can act as reservoirs of human and animal pathogens and play a role in dissemination of infectious agents including antimicrobial-resistant bacteria into the general population, with potential effects on humans and animals. Veterinary personnel also face an inherent risk of zoonotic disease from contact with both healthy and ill animals. All these issues clearly indicate why infection control is an important aspect of veterinary practice. However, the field of veterinary infection control is poorly developed compared to that of infection control in human healthcare, and few resources are currently available to help veterinarians design and implement adequate infection control programs. The objective of this project was to prepare Infection Prevention and Control Best Practices web-based guidelines for use by small animal veterinary clinics. A broad range of topics will be covered in these guidelines, with emphasis on routine practices such as cleaning and disinfection, hand hygiene, personal protective equipment and precautions for zoonotic pathogens. 


\section{Programmed cell death-1 molecule expression by feline dendritic cells as a potential mechanism of immunosuppression during FIV infection}

E. Kuczynski, D. Bienzle

Department of Pathobiology, Ontario Veterinary College

The feline immunodeficiency virus (FIV), like HIV, is a lentivirus in the retrovirus family that causes depletion of CD4+ T lymphocytes and AIDS in cats. The potent antigenpresenting cell, the dendritic cell (DC), may play major roles during early HIV infection, viral spread, and effective antiviral immunity. The dysregulation seen may be in part due to the Programmed cell death-1 (PD-1) pathway. PD-1 (receptor) and PD-L1 (ligand) are found on immune cells, including $\mathrm{T}$ cells and DCs in mice and humans, and feline $\mathrm{T}$ cells. Ligation transmits a negative signal at the $\mathrm{T}$ cell-DC junction that reduces the effector capacity of lymphocytes. Its persistent activation may contribute to reduced anti-HIV immunity. Here, this pathway was studied in the feline immune system. Feline DCs were measured for surface expression and transcript production of PD-1 and PD-L1. Bone marrow samples were collected from healthy out-bred cats, and adherent mononuclear cells were cultured for 8-13 days with cytokines to derive myeloid DCs. Cells were incubated with fluorochrome-linked anti-PD-1 and anti-PDL1 antibodies, and analyzed by flow cytometry. Transcripts were quantified in a real-time PCR assay with PD-1/L1specific primers and DC cDNA as template. Flow cytometry detected mean surface expression of PD-1 on $16.53( \pm 2.52$ SEM)\% and PD-LI on $21.58( \pm 2.01$ SEM)\% of DCs. By one-way ANOVA the difference between PD-1/L1 and control was significant $(p<0.05, \mathrm{n}=6)$. Real-time PCR confirmed production of specific transcripts. Mean expression ratios relative to housekeeping gene $\beta$-actin, were 0.95 ( \pm 0.23 SEM) for PD-1 and 6.54 ( \pm 1.32 SEM) for PD-L1 ( $p<0.05$ by Student's T test, $\mathrm{n}=6$ ). Results indicate that feline DCs express PD-1 and PD-L1, suggesting involvement in immunoregulation and FIV pathogenesis in cats. Future studies will pursue these areas. This research contributes to understanding of immunodeficiency in cats that may be translatable to HIV infection.

\section{Preliminary investigation of the safety and immunogenicity of a Clostridium difficile carbohydrate vaccine in pigs}

T. Wakeford ${ }^{2}$, R. Friendship ${ }^{1}$, S. Weese ${ }^{2}$, M. Monteiro ${ }^{3}$, J. Rousseau $^{2}$

${ }^{1}$ Department of Population Medicine and Department of Pathobiology ${ }^{2}$, Ontario Veterinary College ${ }^{3}$, and Department of Chemistry, College of Physical and Engineering Science

Clostridium difficile is a gram positive, spore forming anaerobic bacterium and is a major cause of enteric disease in pigs. $C$. difficile is common in all ages of pigs, however it can cause severe health problems in piglets born to gilts or multiparous sows 1-7 days after birth. Piglets affected with C. difficile associated disease (CDAD) experience earlyonset scours which can result in reduced productivity, mainly due to decreased weaning weights. $C$. difficile may also be a foodborne disease as it can be found in retail pork. Consequently $C$. difficile has an impact on producers, veterinary practitioners and the swine industry. In this study a carbohydrate vaccine to control the incidence and severity of $C$. difficile associated disease in piglets is explored. The objectives of this study were to evaluate the safety and efficacy of an intramuscular $C$. difficile carbohydrate vaccine in sows and to compare anti- $C$. difficile antibody levels and prevalence of $C$. difficile colonization in piglets from vaccinated versus unvaccinated sows. Ten clinically normal sows that were approximately 30 days from their planned farrowing date were used. Five sows were used as controls and five sows were injected with $400 \mathrm{ug}$ of $C$. difficile capsular polysaccharide. Blood samples were collected from the sows and piglets to test for an immune response and are currently being analyzed by ELISA. Selective culture for $C$. difficile was performed on all fecal samples from the sows and rectal swabs from the piglets. Preliminary results proved that the carbohydrate vaccine was ineffective as there was no evident difference between the piglets of vaccinated sows and unvaccinated sows when testing rectal swabs for $C$. difficile. All 10 litters had piglets test positive for $C$. difficile at the initial two swabbings. At 48-72 hours post farrowing $76 \%$ of the piglets from vaccinated sows and $72 \%$ of piglets from unvaccinated sows tested positive for $C$. difficile. Seven days post farrowing $54 \%$ of the piglets from vaccinated sows and $60 \%$ of piglets from unvaccinated sows tested positive for $C$. difficile. Rectal swabs will continue to be collected from the piglets until they are sent to market. The objective in the longitudinal study is to examine the epidemiological dynamics of $C$. difficile in pigs.

The prevalence of fecal pathogen shedding in calves reared on free access acidified milk versus traditional milk feeding programs in Ontario

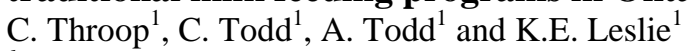

${ }^{I}$ Department of Population Medicine, Ontario Veterinary College

The objective of this cross-sectional study was to compare calves fed free access acidified milk (FAA) to those fed using traditional restricted feeding practices (TR) to determine the prevalence of $C$. parvum oocyst, rotavirus, coronavirus and Escherichia Coli K99 (F5) shedding. Between May and August of 2008, 138 Ontario dairy farms were visited. Fecal samples were collected from calves between the ages of 7 and 30 days and analyzed using the Bio-X fecal pathogen diagnostic test system. To date, 219 samples (FAA=93; TR=126) from 62 herds $(\mathrm{FAA}=26$; $\mathrm{TR}=36$ ) have been analyzed. There were no samples that tested positive for E. Coli K99 (F5). Differences in the 
prevalence of $C$. parvum oocyst (40.9\% vs. $27.0 \%$ for FAA and $\mathrm{TR}$, respectively; $\mathrm{p}=0.14)$ and coronavirus $(8.6 \%$ vs. $3.2 \%$ for FAA and TR, respectively; $\mathrm{p}=0.13$ ) shedding were not statistically significant. There was a tendency for increased prevalence of rotavirus shedding in FAA versus TR $(16.1 \%$ and $7.9 \%$, respectively; $\mathrm{p}=0.06)$. The prevalence of shedding at least one of the 4 pathogens tested was greater for FAA than TR $(40.9 \%$ vs. $27.0 \%$; $\mathrm{p}=0.03)$. After controlling for herd as a random effect, the odds ratio for risk of a FAA calf shedding at least one of the pathogens tested was 1.86 times that for a TR calf, but this finding was not statistically significant $(\mathrm{p}=0.11)$. Preliminary results indicate that the prevalence of $C$. parvum oocyst, rotavirus, and coronavirus shedding is not affected by the type of feeding program utilized.

Expression of uteroglobins and secretory phospholipase $A_{2}$ in the endometrium during normal and compromised early pregnancy in mares E. Traficante ${ }^{1}$, B. N. Lillie ${ }^{2}$, B. A. Quinn ${ }^{2}$, C. Lategan ${ }^{1}$, J. Wenzel $^{1}$, R.O. Waelchli ${ }^{1}$, D. Bienzle ${ }^{2}$, M. A. Hayes ${ }^{2}$, K. J. Betteridge $^{1}$

Departments of Biomedical Sciences ${ }^{1}$ and Pathobiology ${ }^{2}$, Ontario Veterinary College

During the third week of pregnancy in the mare, the encapsulated conceptus is immobilized ("fixed") at the site of subsequent placentation. When pregnancy is compromized by luteolysis induced by administration of an analogue of PGF2aon day 12 or 14 , secretory phospholipase $\mathrm{A}_{2}\left(\mathrm{sPLA}_{2}\right)$ increases in the capsule whereas uteroglobin increases in the uterine secretions. In the present studies, we used qualitative RT-PCR to assess the expression of $\mathrm{SPLA}_{2}$ [EF428565] and three uteroglobin genes, designated UG1, UG2 and UG3, in endometrial tissue obtained by biopsy from normal or PGF2a-compromized pregnancies on Days 15-18 after ovulation. These studies showed that three uteroglobin genes are expressed in the endometrium during the fixation period of normal pregnancy in mares. After administration of PGF2a, endometrial expression of UG3 corresponding to gene 100060053 was higher than in normal pregnancies on Days 15, 16, and 17, whereas expression $\mathrm{sPLA}_{2}$ corresponding to gene was higher on Days 15, 16, 17 and 18. These observations suggest that increases in sPLA2 and UG3 might be involved in failure of early pregnancy in mares.

Opportunities for student involvement in the Canadian Veterinary Reserve

A. Shukla, K. Isnor, D. Kelton

Department of Population Medicine, Ontario Veterinary College

The Canadian Veterinary Reserve (CVR) was launched in 2006 as a joint initiative between the Canadian Food Inspection Agency (CFIA) and the Canadian Veterinary
Medical Association (CVMA). Intended to provide surge capacity in the event of a foreign animal disease (FAD) outbreak or civil emergency, the CVR accepts licensed Canadian veterinary practitioners into the reserve; individuals are then chosen to undergo more extensive CFIA reservist training in Winnipeg in priority sequence. Currently, only licensed veterinarians may apply to the reserve, but the hope is that eventually veterinary students and veterinary technicians may also be eligible to join. The summer of 2008 marked the first year that veterinary students were able to make a real contribution to the reserve, with the creation of the Summer Research Assistantship Positions. These positions have enabled students to undertake a number of research-based tasks to aid the reserve, and the hope is that the positions will be rotated annually among the five Canadian veterinary schools. One of the tasks accomplished during the summer of 2008 included the creation of an annotated bibliography, intended to be accessible to all Canadian veterinary students, highlighting alternative career paths in veterinary medicine. Topics include public health, disaster preparedness and emergency medicine. Other initiatives have been designing a curriculum study to determine whether CVR training may be inserted into the Ontario Veterinary College curriculum, allowing interested parties to graduate as reservists, promoting communication and cooperation with international veterinary reserves, and increasing awareness of the CVR among the veterinary student body.

\section{An evaluation of growth parameters in dairy heifer calves vaccinated at different times in the pre- weaning period}

C. Littlejohn, C. Windeyer, K. Leslie, S. LeBlanc

Department of Population Medicine, Ontario Veterinary College

Growth rate in heifer calves is a key factor affecting the age of sexual maturity and body weight at calving. A myriad of factors contribute to an adequate average daily gain (ADG), such as housing, nutrition, season born, and disease. Bovine respiratory disease is very common in young calves and can significantly impact ADG. Neonatal vaccination programs are not considered to be beneficial, as a result of maternal antibody inhibiting response to the vaccine. However, growing evidence counters this argument. The purpose of this study was to examine the affect of age at vaccination, and number of times vaccinated, on growth in dairy heifer calves. Calves free from disease were enrolled in the study between 1 and 7 days of age. At this time, each calf was randomized into 1 of 4 vaccination groups. Weight, height and temperature measurements were recorded, along with an overall health status score. At days 15-21 and 36-42 the same measurements were taken and a vaccine or placebo was given. A final vaccine was given to all calves between 3 and 4 months of age. The mean weight and height of each treatment group at the four sampling times was calculated 
and no obvious differences existed between the treatment groups. The average weight and height gains were then calculated between both the T2 and T3, and the T3 and T4 intervals. Again, there were no obvious differences. Overall, these preliminary data do not suggest any affect of vaccination regime or age at vaccination on growth rates of dairy heifer calves.

\section{Induction of Cervical Dilation for Artificial Insemination in ewes \\ H.C. Bainbridge and P.M. Bartlewski \\ Department of Biomedical Sciences, Ontario Veterinary College}

The aim of this study was to evaluate the usefulness of Cervidil $^{\circledR}$, a dinoprostone-containing vaginal insert that is currently used to induce cervical ripening and labour in women, as a pre-treatment to enhance the ease of transcervical artificial insemination (AI) in ewes. It was expected that the use of $\mathrm{Cervidil}^{\circledR}$ prior to AI would cause dilation of the cervix, and thus alleviate the difficulty associated with traversing the cervix for semen deposition in ewes. Twelve anestrous Rideau Arcott ewes (May-June) were synchronized into estrus and inseminated with frozenthawed semen using the standardized Guelph AI System. Cervidil $^{\circledR}$ was inserted twelve hours prior to insemination in 6 ewes; six ewes served as untreated controls. Semen was deposited into the uterus in all 6 of the treated ewes, but only in 4 of the 6 control ewes. The time taken to perform the insemination procedure did not differ significantly between the two groups of ewes ( $\mathrm{P}>0.05)$; times ranged from 12-70 seconds in the treated and 30-98 seconds in the control ewes. There was no effect of age and parity of ewes on the time taken to perform AI $(\mathrm{P}>0.05)$. The present results support the hypothesis that Cervidil $^{\circledR}$ may cause cervical dilation facilitating the uterine deposition of semen in the pretreated ewes. The treatment with Cervidil $^{\circledR}$ could potentially be applied to other procedures, such as transcervical embryo transfer and recovery in sheep.

\section{Extra-label drug use on Ontario market sheep farms}

J. Rudniski, C. Moon, L. Scott, P. Menzies, R.J. Reid-Smith, B.P. Avery, N. Janecko, S.A. McEwen

Laboratory for Foodborne Zoonoses, Public Health Agency of Canada, Ontario Veterinary College

Sheep are classified as a minor species in Canada and few drugs are registered for their use, necessitating extra-label drug use (ELDU). ELDU is described by the Veterinary Drug Directorate (VDD), Health Canada as “... refers to the use or intended use of a drug approved by Health Canada in an animal in a manner not in accordance with the label or package insert..." It is believed that ELDU is common in sheep production. This study summarizes the extent and type of ELDU with the goal of providing guidance to the VDD as to drugs and indications to target for minor use minor species (MUMS) approval. This project, which required producers to record all animal treatments for a 12month period, uses a subset of data from a larger project (10 of 49 Ontario sheep farms). Analyses showed that all farms used at least one drug in an extra-label manner. Of all drugs used $(n=59)$, only $30.5 \%$ were prescribed by a veterinarian on at least 1 occasion, and $83.3 \%(n=36)$ of those treatments constituted ELDU. The ELDU level in producer-initiated treatments was also high with a level of $68.2 \%$. Antimicrobials were the most common drug class used and $89.6 \%$ of treatments constituted ELDU. ELDU in Ontario sheep farms is common and may increase the risk of meat and milk residues and possibly antimicrobial resistance due to inappropriate treatment regimes. Streamlining and enhancing the MUMS drug approval process will greatly alleviate this risk.

\section{Characterizing Purkinje cell loss in mice exposed to valproic acid in utero}

S.T. Lang, C.L. Armstrong

Department of Biomedical Sciences, Ontario Veterinary College

Children exposed to valproic acid (VPA) in utero show behavioural and neuroanatomical similarities to children diagnosed with autism. Similarly, rodents exposed to VPA in utero show reduced social and exploratory behaviours as well as developmental deficits in cognitive and motor behaviours mediated by brain structures involved in autism. A consistent finding from post mortem analysis of individuals diagnosed with autism and rodents exposed to VPA in utero is a reduced number of Purkinje cells in the cerebellum. The developmental profile of Purkinje cell loss has yet to be characterized, and may provide insight into the teratogenic mechanism of VPA and the etiology of autism. To accomplish this goal, a combination of wholemount and section immunohistochemistry, using antibodies specific for the calcium binding proteins calbindin and parvalbumin (expressed in all Purkinje cells), was perfomed on cerebellar tissue of postnatal and adult mice exposed to $200 \mathrm{mg} / \mathrm{kg}$ VPA in utero. It was found that acute exposure to VPA in utero affects the integrity of Purkinje cells in specific locations of the cerebellum. The Purkinje cells in the posterior zone (lobules VIII and dorsal lobule IX) and central zone (lobules VI and VII) appear to be more affected than those in the anterior zone (lobules I-V) or the nodular zone (lobule X). Furthermore, Purkinje cell loss is not apparent until after postnatal day ten. These results suggest that a particular subset of Purkinje cells may be preferentially affected following VPA exposure. Additional research needs to be conducted in order to determine the extent of cell death versus calcium binding protein down-regulation, and to investigate the mechanism of action of VPA in the mammalian brain. 
A comparison of feeding behaviour of weaned dairy calves with high, average, and low post-weaning weight gain

K. Hester ${ }^{1}$, A. Stanton ${ }^{1}$, K.E. Leslie ${ }^{1}$, D. Kelton ${ }^{1}$, S.T. Millman $^{1,2}$

${ }^{I}$ Department of Population Medicine, Ontario Veterinary College $e^{2}$,Veterinary Diagnostic and Production Animal Medicine/Biomedical Sciences, Iowa State University

Weaning is a significant transition experienced by dairy calves, involving dietary and other important changes which negatively affect health and welfare. Our objective was to observe feeding behavior of calves following weaning in order to gain insight into the behavioral determinants of weight-gain. Weaning (Day 0) was defined as removal of milk from the diet and movement from individual to group housing. Retrospectively, 55 heifers were divided into three classes based on weight-gain in the first week, with low calves $(n=15)$ in the bottom quartile, high calves $(n=25)$ in the top quartile, and average calves $(n=15)$ in the middle for weight-gain. Behaviour was recorded with time-lapse video recorders and analyzed with 5-min instantaneous scan samples on Days 0, 1, 2, 4 and 6. Three distinct behaviours were identified; consumption, bunk-engaged, and bunkdirected. Differences within days were analysed using univariate analysis with SAS 9.1 software. On Day 0, high calves showed less frequent consumption than average calves $(0.0202 \pm 0.069,0.0529 \pm 0.0071$, respectively, $p<0.05)$. On Day 1, low calves showed less frequent consumption than average and high calves $(0.0535 \pm 0.008,0.0878 \pm 0.0106$, $0.0823 \pm 0.0104$, respectively, $\mathrm{p}<0.05)$. There were no differences in consumption between groups on days 2-6. Overall, consumption and bunk-engaged behaviours increased numerically during the first week, while bunkdirected behaviour reached a maximum on Day 1 and decreased thereafter. Since there were no consistent differences in feeding behavior frequency, factors other than feeding behavior may contribute to weight-gain in dairy calves during the first week post-weaning.

\section{Development of a canine oligomer array comparative genomic hybridization chip}

W. Moore and G. Wood

Department of Pathobiology, Ontario Veterinary College

Cancers often harbour recurrent regions of chromosomal gain and loss, called copy number aberrations (CNA), that contribute to malignant transformation. These regions typically cover areas that contain many genes, making it difficult to identify the specific genes driving tumorigenesis versus the genomic 'noise' inherent in cancers. One technique that detects CNAs is array comparative genomic hybridization (aCGH). This method compares normal DNA to tumour DNA using probes spaced across the entire genome to identify regions that have been deleted or amplified. Currently, there are high resolution oligomer aCGH chips available for mice and humans3, but not dogs. Our goal was to develop an aCGH chip for detection of CNAs in canine cancers, both for use in a clinical setting, as well as for multi-species comparative oncogenomics. Several obstacles were encountered throughout the process of designing the array, including current limitations in genome bioinformatics, canine genome annotation, and probe selection criteria. A beta version of a commercially designed canine array was evaluated and proved inadequate, having too much cross hybridization potential. Our own array is in the design phase, and when complete, we aim to optimize the probes using non-tumour self-self DNA hybridization. In the future, the chip will be used to identify CNAs associated with the development and progression of canine cancers, and to conduct multi-species oncogenomics.

\section{An outbreak of Cysticercus ovis on an Ontario sheep feedlot \\ E. Tatone ${ }^{1}$, P. Menzies ${ }^{2}$, J. Jansen $^{3}$, S. Fernández ${ }^{4}$ and A. S. Peregrine $^{1}$ \\ Departments of ${ }^{1}$ Pathobiology and ${ }^{2}$ Population Medicine, Ontario Veterinary College; ${ }^{3}$ Ontario Ministry of Agriculture, Food and Rural Affairs (OMAFRA); ${ }^{4}$ Organic Agriculture Centre of Canada}

Cysticercus ovis is the larval stage of the tapeworm Taenia ovis. A recent outbreak of $C$. ovis in Ontario threatens the viability of the sheep industry in the province, and potentially Canada. The general public, sheep producers and veterinarians need to be educated about $C$. ovis through the compilation of relevant research and current knowledge. No treatment or vaccine currently exists for $C$. ovis. Therefore, preventing infection is the only option and requires understanding of the parasite life cycle. During the preparation of educational materials for OMAFRA, several issues were uncovered that could inhibit success in the prevention of $C$. ovis. Some of the problems include a lack of traceability of infected sheep, and the inability to release source farm information due to privacy regulations and jurisdiction. The outbreak of $C$. ovis has increased awareness of the parasite and the options for prevention, as well as highlighting some more serious problems that face the sheep industry. Pro-active education of veterinary professionals, veterinary students and sheep producers, via both an informative fact sheet and educational talks, will further increase knowledge and increase pressure on the industry to make the necessary changes to reduce economic loss due to C. ovis. 


\section{A comparison of $\mathrm{Ca}^{2+}$ flux in lymphocytes from calves of various ages}

C. Hoglund, B.A. McBey, K. Chattha, D. Hodgins, P. Shewen

Department of Pathobiology, Ontario Veterinary College

Induction of acquired immunity by vaccination in neonatal calves is much more difficult than older calves. This can be attributed to many factors including immaturity of immune function or blood circulating components. Activation of naïve lymphocytes is a major step in the development of immunity. In this study, we used $\mathrm{Ca}^{2+}$ flux to analyze immune activation potential of lymphocytes from calves of different ages. Lymphocytes from three neonates (2 days, 6 days, 13 days) were compared to three older calves (five months) and challenged with three different doses (5 $\mu \mathrm{g}, 10$ $\mu \mathrm{g}, 15 \mu \mathrm{g}$ ) of Ionomycin, a potent calcium ionophore. Fluorescence activated cell scanning (FACScan) using the fluorochromes Fluo-4 and Fura Red was the preferred method of analysis. Ratiometric calculations comparing the Fluo-4:Fura Red fluorescence intensity ratio post-Ionomycin treatment with the baseline flux (Mean Normalized Ratio) showed that the 2-day old calf had consistently the lowest lymophocyte activation. The five month old calves had the highest activation when challenged with $15 \mu \mathrm{g}$ of Ionomycin. This is a pilot study of the difference in activation in neonatal calves compared to older calves using $\mathrm{Ca}^{2+}$ flux. The small amount of subjects makes it impossible to draw concrete conclusions, but this is an informative beginning. The less intense response that the neonates showed could be imputed to higher levels of corticosteroids or other circulating factors. An improved understanding of age-specific activation levels of lymphocytes could lead to more efficacious vaccination of neonates.

\section{Evaluating the need for standardized reporting in small animal clinical trials}

A Thompson ${ }^{1}$, J. Saint-Onge ${ }^{1}$, R. Elgie ${ }^{1}$, K. Snedeker ${ }^{1}$, J. Valcour $^{2}$, P. Marcynuk ${ }^{2}$, J.Sargeant ${ }^{1,2}$

${ }^{l}$ Centre for Public Health and Zoonoses; ${ }^{2}$ Department of Population Medicine; Ontario Veterinary College

In the field evaluation of the efficacy of therapeutics and preventative strategies, randomized clinical trials are often considered to be the "gold standard". In human clinical medicine, the past two decades have seen numerous studies detailing significant shortcomings in the reporting of important areas of clinical trials. As well, trials conducted with such shortcomings have been associated with biased results in regards to treatment effect. In response to these problems, a group of researchers and journal editors developed the CONSORT (CONsolidated Standards of Reporting Trials) Statement in 2001, whose endorsement by a large number of medical journals has resulted in definite improvement in the reporting of clinical trials. The objectives of this study were to analyze the current state of reporting in veterinary clinical trials, and establish whether key reporting features of these clinical trials are associated with a likelihood of finding positive treatment effects. In order to due this, 100 recent clinical trials in small animal medicine were identified using a structured search, and had their quality assessed using a modified CONSORT checklist by two independent reviewers. To evaluate the associations between quality and outcome, two different reviewers then read the same papers, and extracted data on the specifics of the reported results in terms of significance. Preliminary results show that randomization, blinding and a primary outcome were reported $73 \%, 45 \%$, and $6.4 \%$ of the time, respectively. Additionally, $68 \%$ of articles adequately described signalment and population data, and $85 \%$ described the use of a control group.

\section{Heart rate response in racehorses exhibiting exercise associated arrhythmias}

K. Warnick, M.K.J. McGurrin, and P.W. Physick-Sheard

Departments of Population Medicine and Clinical Studies, Ontario Veterinary College

Sudden death and poor performance are issues of concern for the racing industry, and it is thought that cardiac dysfunction plays a primary role in many cases. A unique study examining heart rate and rhythm of Standardbred horses during live racing took place during the summer and fall of 2007. ECG's were collected using Holter monitors and specially designed girths from 343 individual horse-race events from the time of harnessing to the post-race period. Preliminary visual inspection of data revealed obvious dysrhythmias during recovery in $5 \%$ of horses. We predict that patterns of heart rate response during the race and recovery periods of the sample population will reveal information about the overall stability of heart rate and underlying control mechanisms, and provide insight into possible causal mechanisms in arrhythmia. Analysis of the ECG tracings involves accurately identifying and characterizing each QRS complex. Graphing, time domain, and frequency domain analysis of R-R intervals will be used to explore relationships between beat to beat variation and arrhythmia. When data processing is complete, multivariate modeling will be used to examine the relationship between predictor variables and indices of heart rate stability. This study has allowed us to identify unanticipated arrhythmias, and addresses a problem in equine health that has not previously been examined. If clinical significance is determined, the animal welfare implications will need to be examined in consultation with the racing community. 
The validity of veterinary clinical trials in preharvest food safety: evaluating the need for standardized reporting

J. Saint-Onge, R. Elgie, A. Thompson, P. Marcynuk, J. Valcour, K. Snedeker, J. Sargeant ${ }^{1}$

${ }^{1}$ Centre for Public Health and Zoonoses, Ontario Veterinary College

Clinical trials are fundamental to the practice of evidencebased veterinary medicine. The validity of such trials is central in the application of these results. Biased results can mislead decision making at all levels, from treatment decisions to policy making. In the medical world, a group of scientists and editors worked to develop the CONSORT (CONsolidated Standards of Reporting Trials) statement to address deficiencies in the reporting of clinical trials. Since 2001, CONSORT has been endorsed by many medical journals and has resulted in improved reporting of clinical trials. However, there is little evidence to suggest such standards are practiced in veterinary medicine. Our objectives were to develop a process to evaluate the reporting of pre-harvest food safety clinical trials in order to describe the current status of reporting. Our primary objective was to evaluate whether key components of reporting were associated with finding a positive treatment effect. Using a structured search strategy, 100 clinical trials were randomly selected. A modified CONSORT checklist was used by two independent reviewers to assess the reporting quality of the selected articles. To evaluate associations with outcomes, two reviewers blinded to quality assessment independently extracted outcome data from each trial. Preliminary results indicate that randomization, blinding, and stating a primary outcome were reported 44/100 (44\%), 5/99 (5\%), and 1/90 $(1 \%)$ of the time, respectively. In addition, $52 / 100(52 \%)$ of articles clearly stated the number of animals housed together in each group, 88/100 (88\%) reported interventions with enough detail to replicate them, and 4/100 (4\%) did not use a control (or comparison) group.

\section{Relationship between electrode position and cardioversion energy in transvenous electrical cardioversion}

\section{E. Preiss, P. Physick-Sheard, K. McGurrin, D. Kenney}

Department of Population Medicine, Ontario Veterinary College

Transvenous Electrical Cardioversion (TVEC) is used to treat horses with atrial fibrillation (AF). Electrodes are positioned in the heart and pulmonary artery and shocks are delivered in incremental energies until sinus rhythm is restored or $300 \mathrm{~J}$ maximum energy is reached. The objective of the present study was to evaluate treatment observations, suggesting that precise electrode position has an impact on cardioversion energy. Refined positioning could allow development of TVEC into a standing, outpatient procedure.
Thoracic radiographs from 41 horses treated with TVEC were analyzed. An orthogonal coordinate space was created with zero lying directly beneath the junction of the cranial vena cava and right atrium. Two dimensional, $\boldsymbol{y}$ and $z$ axis, information was collected from radiographs, and custom software was used to determine the range of possible $\boldsymbol{x}$ axis positions that would produce the observed x-ray shadow. Electrodes coordinates were determined, and used to define a 3-D tapered cylinder describing the most direct path for electrical activity to travel between the electrodes. Validity of assumptions made in the software was tested using electrode positions in post mortem casts of the pulmonary arterial tree of catheterized horses, and by theoretical modeling. Multivariate regression analysis revealed sex, weight, and $\boldsymbol{y}$ axis distance between electrodes (main effect and quadratic) to be the only significant terms, explaining $51 \%$ of variation in the model. Electrode positioning strategies employed during the clinical procedures may have influenced these results, however, and further analysis is required before aspects of positioning other than $\boldsymbol{y}$ axis distance can be dismissed.

\section{Expression of interferon- $\delta$ at the conceptus- endometrial interface during the third week of pregnancy in the mare}

J. Wenzel, C. Tayade, S. Cnossen, J. Wessels, N. Linton, B. Quinn, R. Waelchli, M. Hayes, M. Anthony and K. J. Betteridge

Departments of Biomedical Sciences and Pathobiology, Ontario Veterinary College

Interferons have important functions during pregnancy in many species; interferon- $\tau$, for example, is the maternal recognition of pregnancy signal in ruminants. While no equivalent has been found in the horse, evidence suggests that horses do possess a functional gene for interferon- $\delta$ $(\mathrm{IFN}-\delta)$ and that it is expressed at the conceptus-endometrial interface. The present study seeks to further characterize the pattern of IFN- $\delta$ gene expression at the interface in healthy as well as in failing pregnancies, specifically in the bilaminar and trilaminar parts of the yolk-sac wall, and the endometrium. Samples were taken from 16 mares at gestation days 19.5 or 20.5. Half of the samples were from normal pregnancies, and half from pregnancies in which the mares were treated with cloprostenol (an analogue of prostaglandin $\mathrm{F} 2 \alpha$ ) on gestation day 16.5 to induce luteolysis and subsequent pregnancy failure. Total RNA was extracted using RNeasy kit, and complementary DNA was produced by reverse transcriptase PCR. The expression of IFN- $\delta$ mRNA was measured relative to a housekeeping gene, $\beta$ actin, by quantitative real time PCR. Samples analyzed in previous studies, from mares treated with cloprostenol at day 12.5 or 14.5 , showed that mean IFN- $\delta$ expression was increased three days after treatment in both bilaminar yolksac wall and endometrium, and that expression was lower in 
the endometrium than in the yolk-sac wall. The results of this study show that IFN- $\delta$ was expressed in all tissues examined, and that patterns of expression differed in samples collected at 3 days and at 4 days post treatment. Also, expression of IFN- $\delta$ appears to decline in control tissues after day 17 (the period of conceptus fixation). This corresponds to our findings in day 12.5 and 14.5 treated mares. Further research should help to explain the role of IFN- $\delta$ at the conceptusendometrial interface and how it might be involved in maintenance or failure of early pregnancy in mares.

\section{Intracellular aurvival of Staphylococcus aureus small colony variants within bovine cells}

L. Pyne, H. Atalla and B. Mallard

Department of Pathobiology, Ontario Veterinary College

Mastitis is the single most costly disease to dairy farmers. Staph. aureus is a common cause of intra-mammary infections and frequently causes persistent, antibiotic resistant, recurring cases. For these reasons it has recently been proposed that a small colony variant (SCV) subpopulation of S. aureus that has been shown in human medicine to cause antibiotic resistant persistent infections may play an important role in the pathogenesis of $S$. aureus mastitis. A newly identified bovine Staphylococcus aureus SCV was isolated from a herd suffering from chronic mastitis. This strain, Heba3231, the first SCV strain to be isolated from chronic cases of bovine mastitis, was examined to determine its ability to survive intracellularly within bovine aortic endothelial cells (BAECs) as compared to the parent strain (3231) and Newbould 305, a prototypic strain. The strains were added to BAECs in 24 well tissue culture plates and incubated for 1 or 3.5 hours. The extracellular bacteria were killed by the addition of lysostaphin. The amount of SCV recovered from the cell lysate was several folds higher than the parent or prototypic strain at multiplicities of infection of 25 and 100. The parent and prototypic strains were observed to cause greater cell damage than the SCV strain Heba3231. Results indicate an increased ability of the SCV strain to survive within BAECs is likely due to the decreased toxin production characteristic of SCVs. Preliminary data suggest similar results using bovine mammary epithelial cells. Further studies will address the ability of Heba3231 to persist within phagocytes and lymphocytes in order to better understand the role of SCVs in the pathogenesis of mastitis.

\section{Veterinary cardiology online resources \\ J. Webb, M. O'Grady, M.L. O'Sullivan Department of Clinical Studies, Ontario Veterinary College}

The Pattern recognition is an important skill used in diagnosing patients, which requires a clinician to see a condition many times in many different presentations. One of the challenges of teaching students in a clinical setting is that a student's learning experience is limited by the particular cases that come into the clinic during their rotations. Fortunately, most cardiac conditions are diagnosed using diagnostic imaging and other technologies. By having this media accessible online with an accompanying history, students can analyze a case and practice their diagnostic skills at any time. Therefore, to make this information available to students and practitioners, we have created two websites. Vetgo (www.vetgo.com), an educational reference website for veterinary cardiology, provides detailed information on cardiovascular physiology, diseases, and diagnostic and therapeutic techniques. To help users improve their diagnostic skills, it includes tutorials on interpreting radiographs, electrocardiograms (ECG's) echocardiograms, and auscultation. The Radiograph of the Month presents the user with a set of radiographs and a brief history to be used to formulate a diagnosis. The answer reveals a detailed evaluation of the radiograph and a clinical diagnosis. Ocardio (www.ocardio.com) is an online database of cardiology cases and related media. Organized by patient, condition, and procedure, a brief history and diagnosis are provided with media pertaining to the case. Media can include radiographs, ECGs, echocardiogram videos and images, heart sounds, fluoroscope videos, and videos of surgical procedures. The case-based approach allows the user to explore all of the diagnostic modalities and understand how they contribute to making the final diagnosis. Ocardio can also generate a random radiograph, ECG, or heart sound to test the user.

\section{Oral exposure of neonatal pigs to the egg allergen ovomucoid for induction of experimental food allergy \\ K. MacKenzie, P. Rupa, J. Schmied and B.N. Wilkie Department of Pathobiology, Ontario Veterinary College}

Modeling food allergy using outbred animals is an effective tool for characterizing the immunopathogenesis of hypersensitivities and host-environmental factors involved in predisposition to and expression of allergy in humans. An experiment using outbred neonatal swine examined hypersensitivity induction to the chicken egg white protein ovomucoid (Ovm) by oral sensitization. Four litters of Yorkshire piglets were randomly assigned in a split-litter design to one of three treatment groups: intraperitoneally (ip) sensitized/orally challenged, orally sensitized/ip challenged or orally sensitized/orally challenged. Piglets at 14, 21 and 35 days of age received an Ovm/cholera toxin antigen/adjuvant mixture depending on the assigned treatment group. At 46 days of age, piglets were challenged orally with an egg white/yogurt mixture, or ip with Ovm. Piglets in the ip sensitization treatment group were more reliably sensitized and more frequently expressed clinical signs of allergy than those in the oral sensitization groups. Intradermal skin tests and Ovm-specific $\operatorname{IgG}(\mathrm{H}+\mathrm{L})$ and $\mathrm{IgE}$ 
(H) antibody activity were measured by ELISA to confirm sensitization to Ovm. Intraperitoneally sensitized pigs in all four litters were skin test positive; in two litters orally sensitized pigs were found to be skin test positive. Clinical scores were assigned to pigs expressing clinical signs. High variability of expression of clinical signs was observed within and between litters. Although outbred, neonatal pigs remain a potentially suitable model for oral sensitization to food allergens, however, further optimization of the protocol is required to validate its efficacy.

\section{Comparison of Immune Responses in Purebred Holstein and Crossbred Norwegian Red $x$ Holstein Calves and Heifers \\ J.Glauser, S. Cartwright, N. Begley, L. Clark and B. Mallard \\ Department of Pathobiology, Ontario Veterinary College}

The Holstein breed is significantly inbred, and expression of recessive alleles may contribute to poor immune function. Crossbreeding Holsteins to Norwegian Red Friesians may increase heterosis in the Holstein breed. This study compared immune responses of 100 purebred Holstein (HO) to 100 crossbred Norwegian Red x Holstein (NRFX) two to six month old calves and first calf heifers. Calves were immunized with a type 2 antigen, Hen Egg White Lysozyme (HEWL), and were immunized again as first calf heifers in the early post partum period. Calf serum was collected at Day 0 and Day 14 following immunization and heifer serum was collected on Day 0 and Day 7 following reimmunization. The sera were analyzed using Enzyme Linked Immuno-Sorbent Assay (ELISA) to determine antiHEWL IgM and IgG responses in the calves and IgG responses in the heifers. Antibody responses were compared between calves and heifers within and across breeds. Preliminary data from the first 164 calves indicates that NRFX have larger IgM increases than $\mathrm{HO}$ from day 0 to day $14(\overline{\mathrm{x}}=0.0984+/-0.178$ and $\overline{\mathrm{x}}=0.208+/-0.358$, respectively; $\mathrm{P}=0.026)$. This data supports previous studies showing that NRFX have significantly higher primary IgG antibody. Calf IgG responses have a positive linear correlation with calf $\operatorname{IgM}$ responses $(\mathrm{R}=0.7141 ; \mathrm{P}<0.0001)$; however, there is no correlation between calf IgM or IgG responses and secondary IgG responses in the heifers $(R=-0.03339$ and $R=-0.05570$, respectively). More data is required to determine whether these differences associate with improved health.

\section{The diffusion of common cryoprotectant solutes into equine embryos}

S. Moore, T. Chenier

Department of Population Medicine, Ontario Veterinary College

In current reproductive technologies, a reliable method to cryopreserve equine embryos is unavailable. Small equine embryos (<300 um) can be successfully frozen with expected conception rates following transfer of $30-50 \%$. Large equine embryos (>300um) cannot be frozen successfully, with most studies reporting conception rates near $0 \%$. It has been theorized that the large surface area to volume ratio of large embryos or the developing embryonic capsule are to blame. In this research project the diffusion of common cryoprotectant solutes (CPA) into equine embryos $(n=8)$ of 8 and 10 days of age was evaluated by gas chromatography (GC). Six Standardbred mares were super-ovulated with eFSH and artificially inseminated. Embryos were collected on day 8 or 10 post-ovulation. The mares were recycled with an injection of PGF2 and rebred. All embryos were collected over a period of 2 months. Embryos were placed into wells containing either ethylene glycol or glycerol for 30 minutes. Embryos were moved into a dry well, lysed and both the intracellular fluid (ICF) and the trophoblastic tissues sampled for the concentration of CPA by GC. Trophoblastic tissues were homogenized, mixed with 30 ul of PBS then analyzed. Ethylene glycol diffused into all day 8 and day 10 embryos. Glycerol diffused into all day 10 embryos but not all day 8 embryos. More ethylene glycol diffused into day 8 and day 10 embryos than glycerol.

\section{Analysis of DNA integrity of bovine embryos produced with sex sorted sperm \\ L. Crawford ${ }^{1}$, P. Blondin ${ }^{2}$, P. Madan, W. A. King ${ }^{1}$

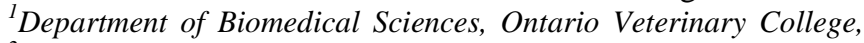 ${ }^{2}$ L'Alliance Boviteq, Saint-Hyacinthe}

Sex sorted semen has significant applications for both research and commercial use, thus optimizing this procedure would prove to be extremely beneficial for agriculture. Bull sperm sorted for sex determination via fluorescence activated cell-sorting (FACS) technology has been shown to be relatively less fertile and produce fewer viable embryos in vitro compared to unsorted controls. Given that DNA can be altered by exposure to ionizing radiation (in this case, ultraviolet light), the objective of this study was to determine if sex sorting resulted in embryos with a higher incidence of DNA damage. It is hypothesized that the FACS procedure negatively affects the DNA of the resulting embryos. Bovine embryos were produced in vitro with sorted and unsorted semen, both frozen and fresh. The quality of embryos was assessed and individual embryos were screened for both double-strand and single-strand DNA breaks using anti- $\gamma$ H2AX immunostaining and TUNEL (terminal deoxynucleotidyl tranferase-mediated dUTP nick end labelling) with subsequent image analysis by confocal microscopy. Anti- $\gamma-\mathrm{H} 2 \mathrm{AX}$ marks double-stranded DNA damage, whereas TUNEL pinpoints DNA fragmentation, characteristic of apoptotic cells. The number of affected cells was determined for each embryo and data was tested for significance using one-way ANOVA. Results suggest that there is no significant difference $(\mathrm{P}>0.001)$ in the number of affected cells between control embryos compared to those 
produced with sex sorted sperm. The results suggest that FACS technology per se does not lead to a higher incidence of DNA damages in embryos. Therefore, further analysis is required to determine the cause of reduced viability and developmental potential among bovine embryos produced from sex sorted semen.

\section{Expression of uteroglobins and secretory phospholipase $A_{2}$ in the endometrium during normal and compromised early pregnancy in mares E. Traficante ${ }^{1}$, B. N. Lillie ${ }^{2}$, B. A. Quinn ${ }^{2}$, C. Lategan ${ }^{1}$, J. Wenzel ${ }^{1}$, R.O. Waelchli ${ }^{1}$, D. Bienzle ${ }^{2}$, M. A. Hayes ${ }^{2}$, K. J. Betteridge $^{\mathrm{a}}$ \\ Departments of Biomedical ${ }^{1}$ Sciences and ${ }^{2}$ Pathobiology, Ontario Veterinary College}

During the third week of pregnancy in the mare, the encapsulated conceptus is immobilized ("fixed") at the site of subsequent placentation. When pregnancy is compromized by luteolysis induced by administration of an analogue of PGF2ạon day 12 or 14 , secretory phospholipase $\mathrm{A}_{2}\left(\mathrm{sPLA}_{2}\right)$ increases in the capsule whereas uteroglobin increases in the uterine secretions. In the present studies, we used qualitative RT-PCR to assess the expression of $\mathrm{SPLA}_{2}$ [EF428565] and three uteroglobin genes, designated UG1, UG2 and UG3, in endometrial tissue obtained by biopsy from normal or PGF2a-compromized pregnancies on Days 15-18 after ovulation. These studies showed that three uteroglobin genes are expressed in the endometrium during the fixation period of normal pregnancy in mares. After administration of PGF2a, endometrial expression of UG3 corresponding to gene 100060053 was higher than in normal pregnancies on Days 15, 16, and 17, whereas expression $\mathrm{sPLA}_{2}$ corresponding to gene was higher on Days 15, 16, 17 and 18. These observations suggest that increases in sPLA2 and UG3 might be involved in failure of early pregnancy in mares.

\section{OVC Digital Library}

M. Brown ${ }^{1}$, N. Lemieux ${ }^{2}$, S. Nykamp ${ }^{3}$

${ }^{1}$ Office of the Dean, ${ }^{2}$ Department of Pathobiology and ${ }^{3}$ Department of Clinical Studies, Ontario Veterinary College

In today's technological world, the internet and computer are commonly used as an educational resource. In order to enhance the quality of education and research at the Ontario Veterinary College, the WebAims MiaSoft database was recently purchased, centralizing the vast resources at OVC and making them available to all members of the college community. The database will allow users to store and easily access files such as images, videos, sound clips, and documents from one common location. The user friendly interface is web-based, making it available anywhere the internet is offered. Access to specific cases or details within a case can be restricted by user groups or specific individuals. User groups will be defined as a general user/student, faculty/staff, submitter or administrator and accounts can be set up with private access for cases used in examinations. The software has an e-learning function, allowing instructors to select a collection of cases for courses, labs or self tests. Users can also select a collection of cases and test themselves by submitting an appropriate diagnosis. These collections can also be restricted with user group access within or outside of the OVC community. The software is compatible with PACs (Picture Archiving and Communication System) filmless radiology system and files from Sapphire (a laboratory information management system) used by the Animal Health Lab, both of which are easily integrated into the database. The database server is housed in the CCS Data Centre with a current storage capacity of $1 \mathrm{~TB}$ and a maximum file size of $40 \mathrm{MB}$. The software is optimized for Windows Internet Explorer 7 and above however, it will work in any browser that supports Flash, CSS-P and JavaScript.

\section{Integrated venous function \\ R. Brill, R. Johnson \\ Department of Biomedical Science, Ontario Veterinary College}

Veins and arteries play significantly different roles in the body. Small changes in venous tone can lead to significant changes in venous return, which ultimately affects cardiac output and blood pressure. Veins and arteries are regulated by a number of different mechanisms including the sympathetic nervous system, local and systemic hormones. The aim of this study was to characterize, using pharmacological and neurogenic activation, the control of venous and arterial function in normal rat mesentery. The effects of the endothelinergic system, potassium chloride (KCL), and neurogenic stimulation in arteries and veins were examined. Mesenteric vessels were isolated from male Sprague-Dawley rats (8-10 weeks old) and changes in vessel diameter were recorded using computer assisted microscopy (Diamtrak $\left(\right.$ ) ). The application of endothelin-1 $\left(10^{-12} \mathrm{M} \rightarrow\right.$ $\left.3 \times 10^{-7} \mathrm{M}\right)$, an ETA and ETB agonist, produced dose dependent contractions in mesenteric veins and arteries, with significantly higher $E_{\max }$ values in the veins $(p<0.05)$. KCL $(20 \mathrm{mM} \rightarrow 120 \mathrm{mM})$ produced a dose-dependent response curve; the response in the veins were larger compared to that of the arteries. Neurogenic stimulation $(0.2 \mathrm{~Hz} \rightarrow 30 \mathrm{~Hz})$ produced a frequency response curve, with contractions appearing greater at lower frequencies in veins compared to the arteries. These results indicate that mesenteric veins are more sensitive to vasoactivation than mesenteric arteries. 


\section{Quantification of rainbow trout (Oncorhynchus mykiss) plasma intelectin}

\section{Edwards, G. M., Lumsden, J. S.}

Department of Pathobiology, Ontario Veterinary College

Two plasma lectins, ladderlectin and intelectin, have been identified in rainbow trout and are postulated to be relevant for innate immunity. Both lectins are known to bind a range of relevant infectious agents. Ladderlectin in plasma has been previously quantified. The purpose of the study was to measure the plasma concentration of intelectin using an indirect capture ELISA. Eighty healthy fish were examined as well as fish infected with bacteria (Aeromonas salmonicida) and fish undergoing an acute phase response following injection with lipopolysaccharide. Intelectin was found to be highly variable in the healthy fish, ranging from $0.12 \mathrm{ng} / \mathrm{mL}$ to $0.52 \mathrm{ng} / \mathrm{mL}$. Groups of fish infected with $A$. salmonicida and those undergoing an acute phase response had increased plasma intelectin but were not significantly different from control groups at most time points. Overall, the natural variation in plasma intelectin concentration is consistent with that of ladderlectin however the biological significance of the variation is unknown. Previous reports in more than one species of fish have indicated that intelectin hepatic mRNA is significantly increased under similar stimuli as used in the present experiments. However, we cannot confirm that intelectin is an acute phase reactant in rainbow trout. Further research will focus on temporal variation in healthy fish and alteration of plasma intelectin on a shorter time scale. 